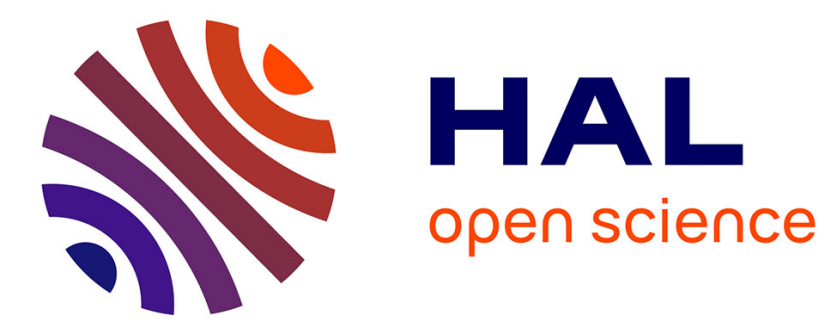

\title{
Les travaux de Robert M. Post sur le kindling (embrasement), la sensibilisation et le conditionnement Georgios (Yorgos) Dimitriadis
}

\section{To cite this version:}

Georgios (Yorgos) Dimitriadis. Les travaux de Robert M. Post sur le kindling (embrasement), la sensibilisation et le conditionnement. Annales Médico-Psychologiques, Revue Psychiatrique, 2012, 10.1016/j.amp.2012.04.002 . hal-01467139

\section{HAL Id: hal-01467139 \\ https://hal.science/hal-01467139}

Submitted on 27 Feb 2017

HAL is a multi-disciplinary open access archive for the deposit and dissemination of scientific research documents, whether they are published or not. The documents may come from teaching and research institutions in France or abroad, or from public or private research centers.
L'archive ouverte pluridisciplinaire HAL, est destinée au dépôt et à la diffusion de documents scientifiques de niveau recherche, publiés ou non, émanant des établissements d'enseignement et de recherche français ou étrangers, des laboratoires publics ou privés. 


\section{Les travaux de Robert M. Post sur le kindling (embrasement), la sensibilisation et le conditionnement}

Yorgos Dimitriadis

\section{Introduction}

Les travaux de Robert M.Post en ont eu des conséquences assez importantes dans la clinique et la thérapeutique psychiatrique actuelle. Du point de vue de l'importance de ses travaux nous rappelons deux faits : 1)Que ses modèles sur le «kindling ${ }^{l}$ » et la sensibilisation ont influencé ses essais cliniques, pionniers dans le domaine -, de l'usage de la carbamazepine dans le trouble bipolaire, premier antiépileptique qui a été étudié et par la suite utilisé dans cette indication.2).Que ses travaux ont été pionniers dans la clinique et la thérapeutique de troubles bipolaires à cycles rapides, le lithium en monothérapie étant réputé peu efficace dans cette indication.

Post part dans son argument d'un fait clinique: «le trouble affectif unipolaire et bipolaire tend pas seulement à être récurent mais aussi à être progressif dans le sens où des épisodes successifs apparaissent après des intervalles de remissions plus courts ou avec une plus grande rapidité de virage» [4]. Il se réfère à plusieurs études statistiques faites à ce propos [3]. Puis il souligne le fait clinique que : «le premier épisode d'un trouble affectif, indépendamment de s'il est maniaque ou dépressif, il est plus probable d'être associé à des stress psychosociaux majeurs que les épisodes qui apparaissent plus tard dans le cours de la maladie » [4]. Il cite même Kraepelin qui, selon cette citation (mise en exergue dans un des ses articles de Post) a observé de

\footnotetext{
${ }^{1}$ Terme introduit en psychiatrie par Robert Post. La traduction du mot « kindling » en Français par « allumage » étant peu élégante nous utiliserons le terme anglais tout au long du texte. Les traductions des extraits d'articles ont été faites par nous même.
} 
faits cliniques semblables.. Partant donc de ces faits cliniques il va tenter de les théoriser de manière neurobiologique en utilisant trois concepts neurobiologiques le kindling, la sensibilisation et le conditionnement ; ces deux deniers concepts sont largement connu en tant que concepts du behaviorisme, le premier de trois, il l'a emprunté à l' épileptologie.

\section{Valeur heuristique de ces concepts pour le trouble bipolaire}

Nous résumons les grosses lignes d'un des articles qu'il a écrit avec ses collaborateurs [3]:

Le « kindling » a été à l'origine décrit par Goddard et ses associés en 1969 en référence à l'éventuel développement de crises majeures d'épilepsie chez les animaux, comme réaction à la stimulation intermittente du cerveau avec du courant électrique qui était initialement inefficace. Le même résultat a pu être produit avec l'administration des agents pharmacologiques. Si le kindling pharmacologique et le kindling électrophysiologique partage quelques caractères temporaux, selon Post et al. Les mécanismes qui les sous-tendent seraient assez différents et concernent des régulations de neurotransmetteurs différents et des chemins neuro-anatomiques différents. Après un certain nombre de répétitions de crises d'épilepsie déclenchées de cette manière, un animal peut avoir de crises d'épilepsie spontanées même en dehors de toute stimulation extérieure. D'autres phénomènes non relatifs directement à des crises motrices d'épilepsie apparaissent dans le processus du kindling, comme une augmentation de l'excitabilité neurale après la stimulation de l'amygdale. Le modèle de kindling pourrait être applicable, selon Post, à des altérations au long cours dans l'excitabilité neurale à des endroits précis du cerveau sans que ce soit nécessaire qu'il y ait production de crises d'épilepsie. Post et al. ont 
étudié un modèle de sensibilisation comportementale, de changement au long cours de la réactivité comportementale après administration répétitive de stimulants psychomoteurs et des agonistes dopaminergiques dans lequel l'application répétitive de petites doses a eu comme résultat une augmentation de l'effet - et non pas de la tolérance - donc un effet de tolérance inverse au fur et à mesure ; une sorte de kindling induit par des substances pharmacologiques avec de l'effet dans le comportement, une sorte de sensibilisation.

La sensibilisation comportementale peut selon Post et al., qui se réfèrent à des études d'autres auteurs à ce sujet, apparaître comme réaction au stress et peut se croiser à des changements produits par des médicaments stimulants (amphétamine, dopamine). Des stress de type, intensité et intermittence particulière pourraient provoquer une sensibilisation comportementale semblable à celle mentionnée plus haut. Post et al. se réfèrent à des travaux de Sklar \& Harris qui ont rapporté que une perte temporaire ou intermittente d' un parent, plutôt qu' une perte continue, était associée avec des plus importantes pathologies dépressive et autres psychopathologies, chez des sujets venant de familles nombreuses. Par ailleurs il note que une exposition à des chocs d'animaux privés de la possibilité de les éviter, a été discutée en tant que model de l'analgésie induite par le stress, la desaide apprise (learned helplessness). Post et al. postulent que il y a des éléments de plus en plus convaincants dans la littérature spécialisée sur le fait que des changements biochimiques induits par le stress venant de l'environnement peuvent être conditionnés et il cite un certain nombre de références bibliographiques sur le sujet, par exemple (Schiff et al). Il avance l'hypothèse que des éléments symboliques d'événements qui ont pu déclenché un épisode dépressif 
pourraient être appris ou conditionnés de façons à ce qu'ils puissent provoquer de la dépression même en l' absence de la stimulation non conditionnée c' est à dire le stress ou la perte réelle. Ils postulent que des stress anticipés ou des pertes imaginées, s'ils sont suffisamment conditionnés, sont éventuellement capables de produire les changement comportementaux, physiologiques et biochimiques qui sont habituellement associés avec un épisode dysthymique. Si, disent-ils, le concept s'avère valide, il se peut qu'il fournisse un pont de transition entre des concepts psychanalytiques relatifs au rôle des pertes réelles ou symboliques et des vues biologiques - endogènes de la dépression. Ainsi il pourrait être expliqué pourquoi on trouve plus des facteurs psychosociaux précipitant plutôt au début que plus tard dans le cours du trouble affectif. Les mécanismes inhérents au conditionnement pourraient offrir une approche conceptuelle de l'émergence d'états apparemment opposés de la dépression (c'est à dire de la manie), suite à des facteurs précipitants similaires. Ils rapportent que Siegel a suggéré que des réponses comportementales, physiologiques et biochimiques opposées à ceux qui ont été initialement induites peuvent de temps en temps apparaître durant le conditionnement. Celui-ci aurait, par exemple, suggéré que des sujets avec un passé d'administration de morphine présentent une réaction de compensation conditionnée quand ils sont confrontés à la procédure habituelle d'administration mais sans la drogue. Ils suggèrent à leur tour que des réponses opposées sur le plan de l'humeur pourraient être développées en tant que des réponses compensatoires qui suivent des épisodes dysthymiques à répétition. De manière analogue, disent-ils, avec les formulations de Siegel des sujet avec une histoire de dépression pourraient présenter des réponses de compensation à 
la dépression, à savoir maniaques, s'ils sont confrontés à des situations qui provoquent habituellement des réactions dépressives, quand seulement le timing, le contexte ou le substrat biologique sont changés.

\section{Développements ultérieurs des mêmes concepts}

Post et al. proposent [4] un mécanisme de transcription de gènes pour expliquer les effets à long terme de ces phénomènes, c'est à dire un mécanisme qui pourrait expliquer la mémoire de la transmission neuronale à long terme. Un tel changement de transcription de gènes est, disent-ils, le proto-oncogène c-fos qui par la suite change l'expression de gènes par sa liaison avec des sites de ADN et l'induction de mRNA et d'autres substances qui peuvent exercer leurs fonctions sur une période des quelques jours à quelques mois. Il rapporte des études selon lesquelles des stress peuvent induire le proto-oncogène c-fos. Ils peuvent induire, selon Post qui se réfère aux études de Kandel (sur l'aplysie) pas seulement des processus biochimiques en tant que procédés d'encodage au long cours mais aussi de changement micro-anatomiques. Plus tard dans le cours du trouble affectif, quand des stress psychosociaux ne sont plus si apparents comme au début, une «mémoire » neurobiologique pour la vulnérabilité aux stress peut fournir un marqueur de trait de longue durée pour l'individu.

Des stress psychosociaux qui ont affaire avec des pertes ou des menaces de perte dans un contexte social pourraient avoir des conséquences cognitives, comportementales et neurobiologiques très différents de celles qui ont affaire avec les menaces de traumatisme physique, qui pourraient être plus pertinentes pour l'induction de syndromes comme le syndrome de stress post-traumatique. Néanmoins quelques uns des mêmes mécanismes de transduction que ceux 
impliqués dans des troubles affectifs, en ce qui concerne les changements au long cours au niveau de transcription de gènes, pourrait être pertinents pour le développement du syndrome de stress post traumatique après une menace sévère touchant éventuellement la vie.

Puis d'un point de vue thérapeutique Post et al, sur la base de la nature séquentielle de l'évolution du trouble affectif, ils ont postulé que la réponse aux traitements médicamenteux pourrait être différente en fonction du stade de l'évolution au long cours. Ils suggèrent que, tandis que la thérapie psychodynamique pourrait être appropriée pour des dysphories mineurs et précoces qui sont liées au stress ou à des épisodes initiaux de dépression majeure, l'usage de thérapies cognitives, interpersonnelles ou comportementalistes pourraient être plus appropriés pour des épisodes récurrents qui commencent à émerger spontanément. Si la maladie est «en automatique », partiellement en raison de plusieurs répétitions d'épisodes, des thérapies cognitives et comportementales qui traitent cette automaticité (qui ciblent l'habitude ou de mécanismes de mémoire striatale) pourraient être plus efficaces que les thérapies dynamiques. De même pour les traitements médicamenteux, il préconise, afin d'éviter le risque de nouveaux épisodes, de commencer plus tôt le traitement et de poursuivre plus long temps la pharmaco-prophylaxie afin d'éviter la transformation «maligne » en cycle rapide et la résistance aux médicaments. Post et al. proposent les mêmes modèles que ceux décrits pour la maladie dépressive et maniacodépressive, comme étant potentiellement des constructions utiles pour divers processus d'adaptation normaux ou pathologiques qui peuvent apparaître du fait de la 
présentation répétée de stress physiologiques, biochimiques ou psychosociaux $[1,2,3,5]$

\section{$\underline{\text { Bibliographie }}$}

1-Post R, Kopanda R. Cocaine, Kindling, and Psychosis. Am. J. Psychiatry. June 1976; 133: (6) 627-634.

2-Post R, Ballenger J. Kindling as a model for alcohol withdrawal syndromes British Journal of Psychiatry, Jul 1978; 133: 1-14.

3-Post R, Rubinov D, Ballenger J. Conditioning and Sensitization in the Longitudinal Course of Affective Illness. British Journal of Psychiatry.1986; 149: 191-201.

4-Post R. Transduction of Psychosocial Stress Into the Neurobiology of Recurrent Affective Disorder. Am.J. Psychiatry. 1992 ; 149: (8) 999-1010. 5-Post R, Weiss S. Kindling versus quenching. Implications for the evolution and treatment of post-traumatic disorder. Ann. N. Y. Acad. Sic. $1997 ; 21$ : 285-295. 